\title{
Incidence and predictors of annual chlamydia testing among 15-29 year olds attending Aboriginal primary health care services in New South Wales, Australia
}

Simon Graham ${ }^{1,2^{*}}$, Rebecca J. Guy ${ }^{1}$, James S. Ward ${ }^{3}$, John Kaldor ${ }^{1}$, Basil Donovan ${ }^{1,4}$, Janet Knox? Debbie McCowen ${ }^{5}$, Patricia Bullen ${ }^{5}$, Julie Booker ${ }^{5}$, Chris O'Brien ${ }^{5}$, Kristine Garrett ${ }^{5}$ and Handan C. Wand

\begin{abstract}
Background: For the past two decades, chlamydia has been the most commonly notified infectious disease among young people (15-29 year olds) in Australia, the United States of America and the United Kingdom and rates have increased annually in these three countries. In Australia, rates of chlamydia are three times higher in Aboriginal compared with non-Aboriginal people. Australian sexually transmissible infection guidelines recommend annual chlamydia testing for 15-29 year old females and males. This analysis will examine the incidence and predictors of annual chlamydia testing in 15-29 year olds attending four Aboriginal Community Controlled Health Services (ACCHS) in Australia.
\end{abstract}

Methods: From 2009-2011, attendance and chlamydia testing data were extracted from the patient system to calculate the number and proportion of 15-29 year olds that were tested for chlamydia and that tested positive for chlamydia by gender (male, female), age-group (15-19, 20-24, 25-29 years), Aboriginal status (Aboriginal, non-Aboriginal people) and by the four ACCHSs sites (1, 2, 3 and 4). A cohort was created to calculate the incidence rate per 100 person-years (PY) and predictors of an annual chlamydia test (a test within 12-months of a previous test/visit) by the above factors using Cox regression. Unadjusted and adjusted hazard ratios (AHR) and their $95 \%$ confidence intervals (Cls) and p-values were calculated with significance at $p<0.05$.

Results: From 2009-2011, there were 2896 individuals who attended the four ACCHSs. Overall , $17 \%$ (22\% of females and $10 \%$ of males) were tested for chlamydia and $9 \%$ tested positive ( $8 \%$ of females and $14 \%$ of males). The median time to an annual chlamydia test was 10.7 months. The cohort included 2318 individuals. Overall the incidence rate of an annual chlamydia test was 9.1 per 100 PY (11.6 in females and 5.8 in males). Predictors of an annual chlamydia test were being female (AHR: 1.7, $95 \%$ Cl: 1.2-2.2, $p<0.01$ ), being 15-19 years old (AHR: 1.6, $95 \%$ Cl: 1.1-2.3, $p<0.01)$ and attending ACCHS site 2 (AHR: 3.8, $95 \% \mathrm{Cl}: 1.8-8.0, p<0.01$ ).

Conclusions: This analysis highlights that opportunistic STI testing strategies are needed to increase annual chlamydia testing in young people; especially males.

Keywords: Indigenous, STI, Sexual health, Quality improvement shimmer

\footnotetext{
* Correspondence: simon.graham@unimelb.edu.au

${ }^{1}$ Kirby Institute, UNSW Australia, Sydney, NSW 2052, Australia

${ }^{2}$ Centre for Epidemiology and Biostatistics, School of Population and Global

Health, University of Melbourne, Melbourne, VIC 3052, Australia

Full list of author information is available at the end of the article
}

(C) 2015 Graham et al. Open Access This article is distributed under the terms of the Creative Commons Attribution 4.0 International License (http://creativecommons.org/licenses/by/4.0/), which permits unrestricted use, distribution, and reproduction in any medium, provided you give appropriate credit to the original author(s) and the source, provide a link to the Creative Commons license, and indicate if changes were made. The Creative Commons Public Domain Dedication waiver (http://creativecommons.org/publicdomain/zero/1.0/) applies to the data made available in this article, unless otherwise stated. 


\section{Background}

Chlamydia is a sexually transmissible infection (STI) and for the past two decades it has been the most commonly notified infectious disease among young people in Australia [1], the United States of America (US) [2], the United Kingdom (UK) [3] and New Zealand [4]. In Australia, chlamydia notification rates are higher in females, 15-29 year olds and those living in regional and remote areas [1]. Chlamydia notification rates are three times higher among Aboriginal and Torres Strait Islanders (hereafter 'Aboriginal') compared with non-Aboriginal people in Australia and rates are increasing $[1,5]$. Higher rates of chlamydia have also been reported among Indigenous compared with non-Indigenous people in Canada, the US and New Zealand [4, 6, 7]. Reasons for these higher rates could be attributed to lower access to primary health care services, and cultural and social determinants of health [8].

An estimated $85 \%$ of chlamydia infections are asymptomatic and as a result regular STI testing is an important prevention strategy to detect and treat infection early, reduce poor health outcomes from developing and to prevent transmission to others [9]. Untreated chlamydia infection can lead to pelvic inflammatory disease and infertility [10, 11]. STI guidelines in the US [2] and the UK [3] recommend annual chlamydia testing of 1624 year old females; however Australian STI guidelines recommend annual chlamydia testing of 15-29 year old females and males [12].

To decrease the prevalence of chlamydia, modelling studies in Australia and the UK have suggested that an annual chlamydia testing rate of $30 \%$ or greater among 15-24 year old females is needed [13, 14]. However, a modelling study conducted in remote Aboriginal communities in Australia, where chlamydia is endemic suggested an annual chlamydia testing rate of $60 \%$ or greater would be required to reduce the prevalence of chlamydia by $9 \%$ [15].

Primary health care centres are recognised as playing an important role in STI testing, treatment and management [16, 17]. In Australia, Aboriginal Community Controlled Health Services (ACCHS) provide culturally appropriate primary health care to Aboriginal people with an estimated 142 ACCHSs across Australia and 42 in the state of New South Wales (NSW) [18]. A community-based survey of 16-30 year old Aboriginal people in NSW found that greater than $50 \%$ of participants preferred to receive STI testing, treatment and education at an ACCHSs compared with non-Aboriginal primary health care services and sexual health clinics [17]. This highlights that ACCHS are the preferred health care provider for young Aboriginal people in NSW.

A cross-sectional study of 16-29 year olds attending non-Aboriginal primary health care centres in Australia found that $8 \%$ were tested for chlamydia, with higher testing rates in females than males ( $12 \%$ vs $4 \%, p<0.01)$ [16]. However this cross-sectional study cannot determine if the same people are tested every year or if different people are being tested. To more accurately examine annual chlamydia testing of the same person, we used a longitudinal study design to estimate the incidence and predictors of an annual chlamydia test among 15-29 year olds who attended four ACCHSs in NSW Australia.

\section{Methods}

\section{The aboriginal population}

In 2014, the Australian Institute of Health and Welfare (AIHW) estimated that there were 713,600 Aboriginal people in Australia which accounted for $3 \%$ of the Australian population [8]. The number of Aboriginal people varied across the Australian jurisdictions with the largest estimated population residing in NSW $(220,902)$ [8]. Nationally, the median age of the Aboriginal population is 21 years compared with 37 years for the nonAboriginal population [8]. Aboriginal compared with non-Aboriginal people have higher rates of chronic and other communicable diseases, higher rates of unemployment; lower levels of home ownership, school completion and life expectancy [8]. To reduce the disparity between Aboriginal and non-Aboriginal people the Australian government has released a number of national strategies including the Fourth National Aboriginal and Torres Strait Islander Blood-Borne Viruses (BBV) and Sexually Transmissible Infections (STI) Strategy, 2014-2017 [19].

This study was conducted prior to the implementation of a sexual health quality improvement (QIP) project commencing in four ACCHSs in NSW known as SHIMMER [20]. SHIMMER aimed to increase STI (chlamydia, gonorrhoea, syphilis, HIV) and hepatitis (hepatitis B and C) testing and improve the management of these infections. To assist in achieving the aims of SHIMMER, this longitudinal analysis was conducted to assist in developing STI testing and management strategies. The QIP used in SHIMMER commenced in March 2012. This analysis focusses on chlamydia because it has the highest prevalence among young Aboriginal people and because we wanted to use chlamydia as a starting point to develop the STI testing and management strategies and then slowly add other testing strategies that would address the other infections.

SHIMMER was conducted in collaboration with four ACCHSs in NSW to ensure Aboriginal people were included in the decision making process, maintained ownership of their data and were integral to the implementation of the SHIMMER project. The four ACCHSs varied in size and scope of medical, allied health and health education services provided to local Aboriginal communities. They were all located 
in regional areas of NSW and serviced different Aboriginal communities. The four ACCHSs were located large distances from each other to limit the possibility of young people attending multiple ACCHSs. They also had independent management and protocols for medical and allied health services provided to the local Aboriginal communities.

\section{Data extraction}

A data extraction tool called the 'GeneRic Health Network Information Technology for the Enterprise' $\left(\right.$ GRHANITE $\left.^{\mathrm{Tm}}\right)$ [21] was installed onto the electronic patient system at the four ACCHSs. GRHANITE ${ }^{\mathrm{m}}$ extracted de-identified patient attendance and STI testing data through the internet. Only medical consultations with a doctor or nurse were included, with allied health consultations such as dental and physiotherapy excluded.

\section{Statistical analysis}

Data from 2009-2011 were used to calculate the following among $15-29$ year olds:

1. The number and proportion of individuals that attended (attendance) by the following factors:

a. Gender (female, male)

b. Age-group (15-19, 20-24, 25-29 years)

c. Aboriginal status (Aboriginal, non-Aboriginal)

d. ACCHS site (1,2,3 and 4)

2. The number and proportion of individuals who were tested for chlamydia by the above mentioned factors (repeat visits and tests of the same person were remove - proportion tested overall).

3. The number and proportion of individuals who tested positive overall by the above mentioned factors (repeat positives and tests of the same person were removed -positivity).

4. The proportion of individuals who were tested at least once for chlamydia in each year (in 2009 and in 2010 and in 2011) by gender (repeat tests and visits of the same person within a 12-month period were removed) - proportion tested annually).

We used a chi-squared test with statistical significance at $p<0.05$ to assess if there were any differences in chlamydia testing in females and males by the above mentioned factors

We then created a cohort to assess the incidence rate of an annual chlamydia test and predictors of an annual chlamydia test from 2009-2011. An annual chlamydia test was defined as a test within 12months of a previous test/visit. Those aged 15-29 years entered the cohort at their first chlamydia test in 2009 or if not tested in 2009, then those who attended from 1st January 2010. We selected this date because national STI guidelines in Australia recommend annual chlamydia testing of 15-29 year olds [12] and to ensure twelve-months had passed since their previous chlamydia test. If a person was 29 years old when they entered the cohort in 2009 or 2010, they stayed in the cohort until they were tested even if they turned 30 year olds.

Using this cohort we calculated the following among 15-29 year olds:

1. The incidence rate of annual chlamydia testing per 100 person-years (PY) overall and by the above mentioned factors and by Year (in 2010 and in 2011 - incidence rate of annual chlamydia testing)

2. The predictors of annual chlamydia testing by the above mentioned factors - predictors of annual chlamydia testing)

To examine the predictors of annual chlamydia testing we used univariate and multivariate Cox regression. The regression analysis was restricted to the variables that were collected by GRHANITE ${ }^{\mathrm{TM}}$. Unadjusted and adjusted hazard ratios (HR) and their $95 \%$ confidence intervals (CIs) and $\mathrm{p}$-values were calculated with statistical significance at $p<0.05$.

Data were analysed using STATA 12 statistical software (STATA Corporation, College Station TX).

\section{Ethical approval}

The SHIMMER project received ethical approval from the Aboriginal Health \& Medical Research Council of NSW and the University of New South Wales Human Research Ethics Committee. We also received approval from each participating ACCHSs Boards and signed agreements with each ACCHSs detailing issues such as confidentiality, privacy, consent, use of patient data, publications, and roles and responsibilities. The SHIMMER project was funded by the NSW Ministry of Health.

\section{Results}

\section{Attendance}

From 2009-2011, 2896 individuals aged 15-29 years attended the four ACCHSs. A higher proportion were aged $15-19$ years $(38 \%)$, Aboriginal $(81 \%)$ and attended ACCHSs site 3 (41 \%, Table 1$)$.

\section{Proportion tested overall}

Overall, $17 \%$ of individuals were tested for chlamydia, $22 \%$ of females and $10 \%$ of males $(p<0.01$, Table 1$)$. Among males, a higher proportion of 15-19 year olds (12\%) were tested for chlamydia compared with the other two age-groups; however this was not statistically significant $(p=0.14)$. A higher proportion of Aboriginal 
Table 1 Number and proportion tested for chlamydia and positivity in 15-29 year olds, 2009-2011

\begin{tabular}{|c|c|c|c|c|}
\hline \multirow[t]{2}{*}{$\begin{array}{l}\text { Demographic factors } \\
\text { and ACCHS site }\end{array}$} & Individuals & $\begin{array}{l}\text { Tested for } \\
\text { chlamydia }\end{array}$ & $P$-value* & Tested positive \\
\hline & n (\%) & n (\%) & & n (\%) \\
\hline \multirow[t]{3}{*}{ Overall } & $2896(100)$ & 495 (17) & & $47(9)$ \\
\hline & & Males & & \\
\hline & $1223(42)$ & $119(10)$ & $<0.01$ & $17(14)$ \\
\hline \multicolumn{5}{|l|}{ Age-group (years) } \\
\hline $15-19$ & $481(39)$ & $56(12)$ & 0.14 & $9(16)$ \\
\hline $20-24$ & $354(29)$ & $33(9)$ & & $4(12)$ \\
\hline $25-29$ & $388(32)$ & $30(8)$ & & $4(13)$ \\
\hline \multicolumn{5}{|l|}{ Aboriginal status } \\
\hline Aboriginal & $1023(84)$ & $110(11)$ & $<0.01$ & $15(14)$ \\
\hline Non-Aboriginal & $200(16)$ & $9(5)$ & & $2(22)$ \\
\hline \multicolumn{5}{|l|}{ ACCHS site } \\
\hline 1 & $220(18)$ & $5(2)$ & $<0.01$ & $0(0)$ \\
\hline 2 & $178(15)$ & $27(15)$ & & $4(15)$ \\
\hline 3 & $514(42)$ & $77(15)$ & & $12(15)$ \\
\hline \multirow[t]{3}{*}{4} & $311(25)$ & $10(3)$ & & $1(10)$ \\
\hline & & Females & & \\
\hline & $1673(58)$ & $376(22)$ & & $30(8)$ \\
\hline \multicolumn{5}{|l|}{ Age-group (years) } \\
\hline $15-19$ & $605(36)$ & $130(21)$ & 0.07 & $20(15)$ \\
\hline $20-24$ & $578(35)$ & $148(26)$ & & $8(5)$ \\
\hline $25-29$ & $490(29)$ & $98(20)$ & & $2(2)$ \\
\hline \multicolumn{5}{|l|}{ Aboriginal status } \\
\hline Aboriginal & $1323(79)$ & $316(24)$ & $<0.01$ & $27(9)$ \\
\hline Non-Aboriginal & $349(21)$ & $60(17)$ & & $3(5)$ \\
\hline \multicolumn{5}{|l|}{ ACCHS site } \\
\hline 1 & $402(24)$ & $16(4)$ & $<0.01$ & $3(18)$ \\
\hline 2 & $204(12)$ & $42(20)$ & & $5(12)$ \\
\hline 3 & $673(40)$ & $249(37)$ & & $17(7)$ \\
\hline 4 & 394 (24) & $69(17)$ & & $5(7)$ \\
\hline
\end{tabular}

*p-value with significance at $p<0.05$

compared with non-Aboriginal males were tested (11\% vs $5 \%, p<0.01)$. Among females, a higher proportion of 20-24 year olds (26\%) were tested for chlamydia compared with the other two age-groups (Table 1); however this was not statistically significant $(p=0.07)$ and a greater proportion of Aboriginal females compared with non-Aboriginal females were tested (24\% vs $17 \%, p<0.01)$.

\section{Proportion tested annually}

The proportion of individuals tested for chlamydia in each year of the study was similar; from $7 \%$ in 2009 to $6 \%$ in 2010 and $8 \%$ in 2011. Among females, $9 \%$ were tested in 2009, $10 \%$ in 2010 and $10 \%$ in 2011and among males, 3 \% were tested in 2009, 2 \% in 2010 and $7 \%$ in 2011.

\section{Incidence rate of an annual chlamydia test}

A total of 2318 individuals were included in the cohort and the median time from entering the cohort to a chlamydia test was 10.7 months. Overall, the incidence rate of an annual chlamydia test per 100PY was 9.1; higher in females, 20-24 year olds, Aboriginal people and those who attended ACCHSs site 3 (Table 2). Among females and males, the highest rate of an annual chlamydia test was in 20-24 year olds (Figs. 1 and 2).

\section{Predictors of annual chlamydia testing}

Independent predictors of an annual chlamydia test were being female, being 15-19 year olds, and attending ACCHS site 2 (Table 2).

\section{Positivity}

Over the three-year period, there were 495 individual chlamydia tests with $9 \%$ testing positive (Table 1). Although a higher number of positive chlamydia tests were in females than males (30 vs 17 ), a higher proportion of males than females tested positive ( $14 \%$ vs $8 \%$, Table 1$)$. Among males, the highest positivity was in 15-19 year olds (16\%) compared with the other two age-groups and in females, positivity was highest in 15-19 year olds (15\%, Table 1).

\section{Discussion}

This study found that $17 \%$ of young people were tested for chlamydia over a three-year period and $9 \%$ had an annual chlamydia test. Although a higher number of females than males tested positive for chlamydia a higher proportion of males than females tested positive. Independent predictors of an annual chlamydia test were being female, being 15-19 years old and attending ACCHS site 2 .

A strength of this study was that it examined chlamydia testing by using routinely collected attendance and STI testing data from the electronic patient system at four ACCHSs which provided a large sample size for the analysis. There are a number of limitations of our study including; attendance and chlamydia testing data that were not entered into the electronic patient record system were excluded. It is possible that some individuals may have undergone repeat testing at nonAboriginal primary health care centres; therefore we may have under-estimated annual testing rates. The regression analysis did not include the testing of other STIs which could have been a factor in predicting chlamydia testing. GRHANITE ${ }^{\mathrm{TM}}$ could not extract the reason why young people attended the ACCHS and this 
Table 2 Incidence rates and predictors of chlamydia testing in 15-29 year olds, 2009-2011

\begin{tabular}{|c|c|c|c|c|c|}
\hline $\begin{array}{l}\text { Demographic factors, year and } \\
\text { ACCHS site }\end{array}$ & Incidence rate ${ }^{\mathrm{a}}(95 \% \mathrm{Cl})^{\mathrm{b}}$ & Unadjusted hazard ratio $(95 \% \mathrm{Cl})^{\mathrm{b}}$ & $p$-value & Adjusted hazard ratio $(95 \% \mathrm{Cl})^{\mathrm{b}}$ & $p$-value* \\
\hline $2009-2011$ & $9.1(8.1-10.3)$ & & & & \\
\hline \multicolumn{6}{|l|}{ Year } \\
\hline 2010 & $9.6(7.8-11.8)$ & & & & \\
\hline 2011 & $8.9(7.7-10.3)$ & & & & \\
\hline \multicolumn{6}{|l|}{ Gender } \\
\hline Female & $11.6(10.1-13.3)$ & $1.5(1.1-1.9)$ & $<0.01$ & $1.7(1.2-2.2)$ & $<0.01$ \\
\hline Male & $5.8(4.6-7.3)$ & 1 & - & 1 & - \\
\hline \multicolumn{6}{|l|}{ Age-group (years) } \\
\hline $15-19$ & $9.0(7.4-11.0)$ & $1.3(0.9-1.8)$ & 0.06 & $1.6(1.1-2.3)$ & $<0.01$ \\
\hline $20-24$ & $11.0(9.1-13.2)$ & $1.2(0.9-1.6)$ & 0.24 & $1.3(0.9-1.8)$ & 0.11 \\
\hline $25-29$ & $7.1(5.6-9.1)$ & 1 & & 1 & \\
\hline \multicolumn{6}{|l|}{ Aboriginal status } \\
\hline Aboriginal & $9.7(8.6-11.1)$ & $1.1(0.8-1.6)$ & 0.50 & $1.2(0.9-1.8)$ & 0.05 \\
\hline Non-Aboriginal & $6.3(4.5-8.8)$ & 1 & - & 1 & - \\
\hline \multicolumn{6}{|l|}{ ACCHS site } \\
\hline 1 & $1.1(0.5-2.2)$ & $1.6(0.7-3.5)$ & 0.25 & $1.6(0.7-3.6)$ & 0.22 \\
\hline 2 & $13.6(10.4-17.8)$ & $3.4(1.6-7.1)$ & 0.01 & $3.8(1.8-8.0)$ & $<0.01$ \\
\hline 3 & $14.0(12.0-16.4)$ & $2.0(0.9-4.4)$ & 0.07 & $2.2(0.9-4.7)$ & 0.05 \\
\hline 4 & $7.6(5.9-9.8)$ & 1 & & 1 & \\
\hline
\end{tabular}

${ }^{\mathrm{a}}$ Incidence rate per 100 person-years, ${ }^{\mathrm{b}} \mathrm{Hazard}$ ratio and $95 \%$ confidence interval, ${ }^{*} p$-value - significance at $p<0.05$

could have influenced the predictors of chlamydia testing. We were not able to identify those individuals who were residents of the local Aboriginal communities compared with visitors to the Aboriginal communities; which could result in our study under-estimating annual chlamydia testing rates as local residents are able to return annually to be tested compared to visitors to the communities.

In our study, the incidence rate of an annual chlamydia test in females was 11.6 per 100PY. Two other longitudinal studies have examined the incidence rate of an annual chlamydia test in females; one in the US and another in Australia [22, 23]. The US study found that the incidence rate of an annual chlamydia test ranged from 2.1 per 100 woman-years in 15-19 year old females to 13.5 per 100 woman-years in 25 year old females [22] and the Australian study found the incidence rate of an annual chlamydia test was 4.4 per 100 woman-years in 16-29 year old females attending primary health care centres and sexual health clinics [23]. Overall, the annual testing rate in our study was higher compared with the US and other Australian study.

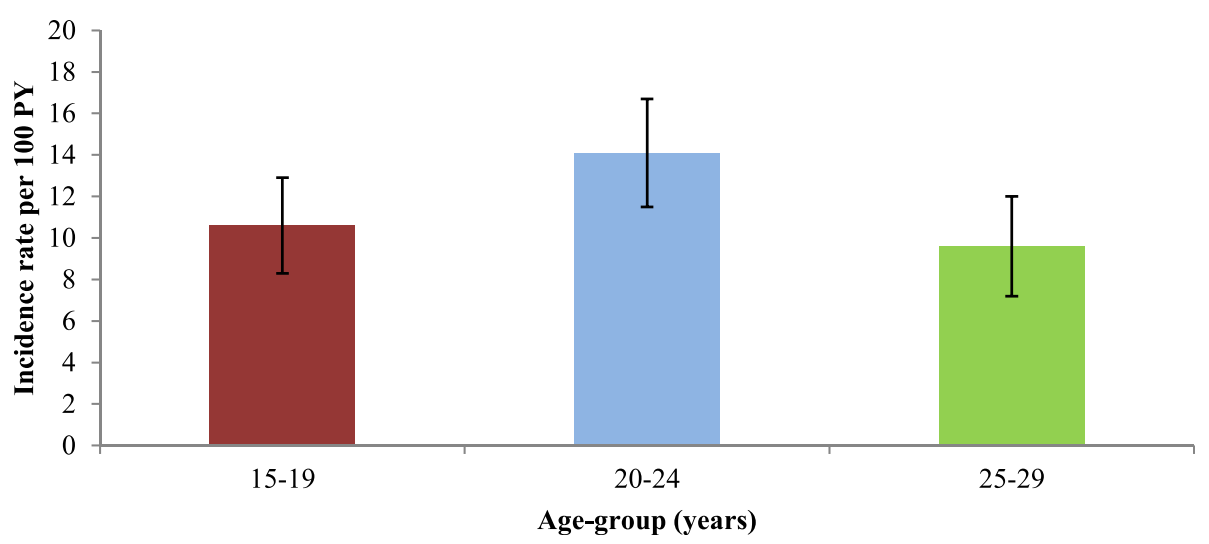

Fig. 1 Incidence rate and $95 \%$ confidence intervals of chlamydia testing in females by age-group, 2009-2011 


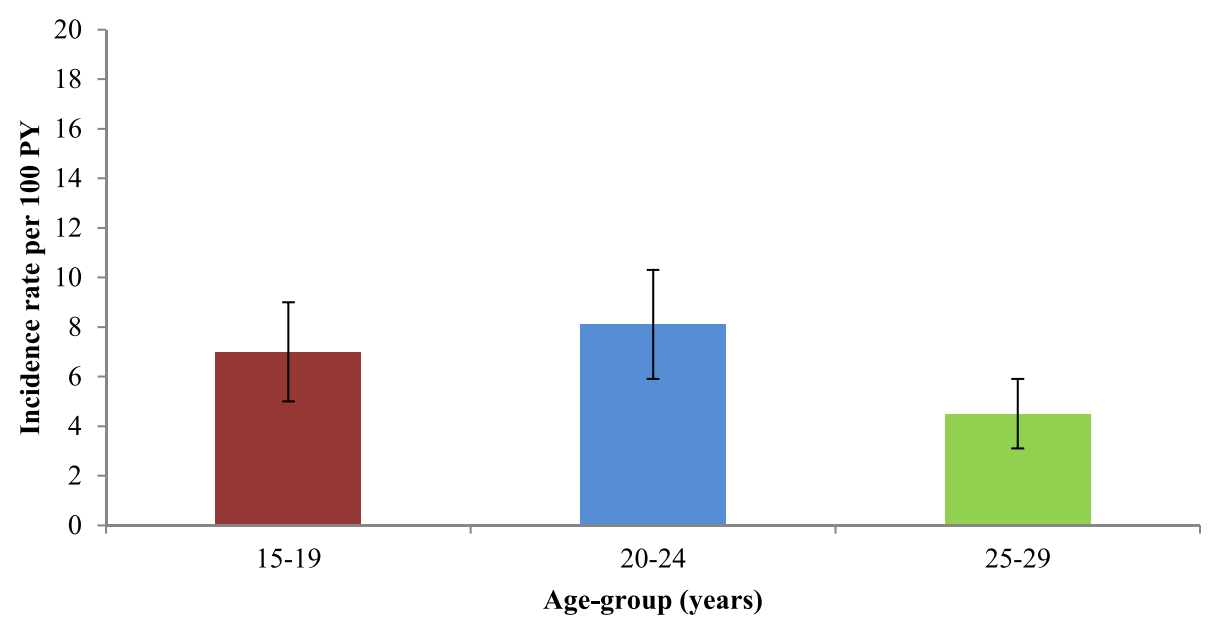

Fig. 2 Incidence rate and 95 \% confidence intervals of chlamydia testing in males by age-group, $2009-2011$

Being female was an independent predictor of having an annual chlamydia test in our study. Higher chlamydia testing rates in females compared with males has been a common finding in a number of other studies [16, 24]. One factor influencing this could be the types of consultations that females are having, such as reproductive health consultations (contraception, Pap smear, pregnancy). A study conducted in primary health care centres in Australia found that reproductive health consultations were highly associated with chlamydia testing [16]. To increase chlamydia testing in males the ACCHS could include chlamydia testing in adult health checks (AHCs) if not already included. AHC are a general health assessment offered in ACCHS [25]. For every completed AHC the ACCHS can receive $\$ 212$ from the Australian government's Medicare Benefits Scheme. A completed AHC can be charged every nine-months [25]. A clinical audit was undertaken in the SHIMMER project which highlighted that $50 \%$ of AHCs among young males included a chlamydia test; however only $16 \%$ of the males were offered an AHC [26]. Offering more AHC could be an effective STI testing strategy to increase chlamydia testing in young males and among females who are not attending for reproductive health reasons.

In our study, $9 \%$ of 15-29 year olds had an annual chlamydia test. The importance of regular chlamydia testing is highlighted by three factors; that chlamydia re-infection is common ( 22\%) [27], that $85 \%$ of chlamydia infections are asymptomatic [9] and that among those females who had untreated chlamydia infection, $10 \%$ developed pelvic inflammatory disease with 12-months [28]. As chlamydia re-infection is common annual chlamydia testing is an important prevention strategy with Australian STI guidelines recommending a re-test for chlamydia three-months after being diagnosed [12]. These factors highlight the importance of developing STI testing strategies for young people that will identify new infections early and provide treatment and education.

\section{Conclusions}

This analysis highlights that opportunistic STI testing strategies could be used by the ACCHSs to increase annual chlamydia testing in young attendees; especially males.

\section{Abbreviations \\ Aboriginal: Aboriginal and Torres Strait Islander; ACCHS: Aboriginal community controlled health services; AlHW: Australian Institute of Health and Welfare; AHC: Adult health check; BBV: Blood borne viruses; Cls: $95 \%$ confidence intervals; GRHANITE TM: Generic Health Network Information Technology for the Enterprise; HR: Hazard ratio; NSW: New South Wales; PY: Person-years; QIP: Quality improvement program; STI: Sexually transmissible infection; SHIMMER: Sexual health quality improvement program; UNSW: University of New South Wales; UK: United Kingdom; US: United States of America.}

\section{Competing interests}

The authors declare that they have no competing interests.

\section{Authors' contributions}

SG, RG, HCW and JMK conceived and developed the idea for the analysis. SG conducted the statistical analyses, interpretation of the results, wrote the first and subsequent drafts and submitted the manuscript. RG, HCW and JMK contributed to the design of the longitudinal cohort, the statistical analyses, writing the manuscript and interpretation of results. BD, JK, JW, DM, PB, JB, $\mathrm{CO}$ and KG contributed to the writing of the manuscript and interpretation of results. All authors have read and approved the final version of the manuscript.

\section{Authors' information}

Not applicable.

\section{Acknowledgements}

Simon Graham, Julie Booker, Chris O'Brien, Kristine Garrett and James Ward are Aboriginal Australians and acknowledge the contribution Aboriginal and Torres Strait Islander Australians make to this work. We would like to acknowledge all the staff at each ACCHS who provide culturally appropriate medical, allied health and education to improve the health and well-being of Aboriginal people. We would like to acknowledge the investigators of the STRIVE project a large sexual health QIP project implemented in remote 
Aboriginal communities. The GRHANITE ${ }^{\mathrm{TM}}$ data extraction tool was developed by $\operatorname{Dr}$ Douglas Boyle at the University of Melbourne. James Ward and Simon Graham were the principal investigators for the SHIMMER project. The SHIMMER project was funded by the New South Wales Ministry of Health. The Kirby Institute is affiliated with the Faculty of Medicine, UNSW Australia. Simon Graham is supported by a McKenzie Post-doctoral fellowship and by the Melbourne Poche Centre for Indigenous Health at the University of Melbourne. Rebecca Guy, John Kaldor and Basil Donovan are supported by National Health and Medical Research Council Fellowships.

\section{Author details}

${ }^{1}$ Kirby Institute, UNSW Australia, Sydney, NSW 2052, Australia. ${ }^{2}$ Centre for Epidemiology and Biostatistics, School of Population and Global Health, University of Melbourne, Melbourne, VIC 3052, Australia. ${ }^{3}$ South Australian Health and Medical Research Institute, Adelaide, SA 5000, Australia. ${ }^{4}$ Sydney Sexual Health Centre, Sydney Hospital, Sydney, NSW 2000, Australia. ${ }^{5}$ Aboriginal Community Controlled Health Service, New South Wales, NSW, Australia.

Received: 14 January 2015 Accepted: 24 September 2015 Published online: 30 September 2015

\section{References}

1. Kirby Institute. Bloodborne viral and sexually transmitted infections in Aboriginal and Torres Strait Islander people: Annual Surveillance Report. Kirby Institute, University of New South Wales. 2015. kirby.unsw.edu.au/ surveillance/2015-aboriginal-surveillance-report-hiv-viral-hepatitis-stis. Assessed on 26 August 2015.

2. Centers for Disease Control and Prevention United States Department of Health and Human Services. Sexually Transmitted Diseases Treatment Guidelines, 2010. Centers for Disease Control and Prevention United States Department of Health and Human Services. MMWR. 2010;59:12.

3. Public Health England. Infection report: Sexually transmitted infections and chlamydia screening in England. vol. 8: Health Protection Report, Public Health England. 2014.

4. New Zealand Ministry of Health. Epidemiology in New Zealand. Chlamydia Management Guidelines. New Zealand Ministry of Health. 2008 www.bpac.org.nz/resources/handbook/sti/sti.asp. Assessed on 26 August 2015.

5. Graham S, Guy RJ, Donovan B, McManus H, Su JY, El-Hayek C, et al. Epidemiology of chlamydia and gonorrhoea among Indigenous and non-Indigenous Australians, 2000-2009. Med J Aust. 2012;197(11):642-6.

6. Centers for Disease Control and Prevention. 2013 Sexually Transmitted Diseases Surveillance. In.: National Center for HIV/AIDS, Viral Hepatitis, STD and TB Prevention, Centers for Disease Control and Prevention. 2014.

7. Wardman D, Quantz D, Clement K. HIV/AIDS: testing and risk behaviors among British Columbia's rural Aboriginal population. Int J Circumpolar Health. 2006;65(4):313-21.

8. Australian Institute of Health and Welfare. The health and welfare of Australia's Aboriginal and Torres Strait Islander peoples. Canberra: Australian Institute of Health and Welfare; 2015. http://www.aihw.gov.au/publicationdetail/?id=60129550168. Assessed on 26 August 2015.

9. Korenromp EL, Sudaryo MK, de Vlas SJ, Gray RH, Sewankambo NK, Serwadda $D$, et al. What proportion of episodes of gonorrhoea and chlamydia becomes symptomatic? Int J STD AIDS. 2002;13(2):91-101.

10. Haggerty CL, Gottlieb SL, Taylor BD, Low N, Xu F, Ness RB. Risk of sequelae after Chlamydia trachomatis genital infection in women. J Infect Dis. 2010;201 Suppl 2:S134-55.

11. Westrom L, Joesoef R, Reynolds G, Hagdu A, Thompson SE. Pelvic inflammatory disease and fertility. A cohort study of 1,844 women with laparoscopically verified disease and 657 control women with normal laparoscopic results. Sex Transm Dis. 1992;19(4):185-92.

12. National Aboriginal Community Controlled Health Organisation and the Royal Australian College of General Practitioners: Sexual health and bloodborne viruses. National guide to a preventative health assessment for Aboriginal and Torres Strait Islander people, 2nd edition. 2012. National Aboriginal Community Controlled Health Organisation and the Royal Australian College of General Practitioners.http://www.naccho.org.au/ download/aboriginal-health/1.National\%20guide\%20to\%20a\%20 preventive $\% 20$ health\%20assessment\%20for\%20Aboriginal\%20and\%20 Torres\%20Strait\%20lslander\%20people\%20(2).pdf. Accessed on 26 August 2015.
13. Regan DG, Wilson DP, Hocking JS. Coverage is the key for effective screening of Chlamydia trachomatis in Australia. J Infect Dis. 2008;198(3):349-58.

14. Turner KME, Adams EJ, LaMontagne DS, Emmett L, Baster K, Edmunds WJ. Modelling the effectiveness of chlamydia screening in England. Sex trans infect. 2006;82(6):496-502.

15. Hui BB, Wilson DP, Ward JS, Guy RJ, Kaldor JM, Law MG, et al. The potential impact of new generation molecular point-of-care tests on gonorrhoea and chlamydia in a setting of high endemic prevalence. Sex Health. 2013;10(4):348-56.

16. Kong FY, Guy RJ, Hocking JS, Merritt T, Pirotta M, Heal C, et al. Australian general practitioner chlamydia testing rates among young people. Med J Aust. 2011;194(5):249-52.

17. Ward J, Bryant J, Worth H, Hull P, Solar S, Bailey S. Use of health services for sexually transmitted and blood-borne viral infections by young Aboriginal people in New South Wales. Aust J primary health. 2013;19(1):81-6.

18. Aboriginal Health \& Medical Research Council of New South Wales. Who we are: Annual Report 2013-2014. Aboriginal Health \& Medical Research Council of New South Wales. http://www.ahmrc.org.au/index.php?option=com_ docman\&task=cat_view\&gid=29\&ltemid=45. Accessed 26 August 2015.

19. Department of Health and Aging. Fourth National Aboriginal and Torres Strait Islander Blood-Borne Viruses and Sexually Transmissible Infections Strategy 2014-2017. Canberra: Department of Health and Aging; 2014. http://www.health.gov.au/internet/main/publishing.nsf/Content/ohp-bbvsatsi. Assessed on 26 August 2015.

20. Graham S, Wand HC, Ward JS, Knox J, McCowen D, Bullen P, et al. Attendance patterns and chlamydia and gonorrhoea testing among young people in Aboriginal primary health centres in New South Wales, Australia. Sex Health 2015. http://dx.doi.org/10.1071/SH15007.

21. Boyle DIR, Kong F. A Systematic Mechanism for the Collection and Interpetation of Display Format Pathology Test Results from Australian Primary Care Records. eletronic Journal of Health Informatics (eJHI). 2011;6(2):e18.

22. Heijne JC, Tao G, Kent CK, Low N. Uptake of regular chlamydia testing by U.S. women: a longitudinal study. Am J Prev Med. 2010;39(3):243-50.

23. Walker J, Tabrizi SN, Fairley CK, Chen MY, Bradshaw CS, Twin J, et al. Chlamydia trachomatis Incidence and Re-Infection among Young Women Behavioural and Microbiological Characteristics. Plos ONE. 2012;7(5).

24. Ward J, Goller J, Ali H, Bowring A, Couzos S, Saunders M, et al. Chlamydia among Australian Aboriginal and/or Torres Strait Islander people attending sexual health services, general practices and Aboriginal community controlled health services. BMC Health Serv Res. 2014;14:285.

25. Commonwealth of Australia's Department of Health and Ageing. Aboriginal and Torres Strait Islander health assessments (MBS item 715). Closing the Gap: Tackling Indigenous chronic disease. http://www9.health.gov.au/mbs/ fullDisplay.cfm?type=note\&q=A33\&qt=notelD\&criteria=715 Accessed on 26 August 2015.

26. Graham S, Guy RJ, Wand HC, Kaldor J, Donovan B, Knox J, et al. A sexual health quality improvement program (SHIMMER) triples chlamydia and gonorrhoea testing rates among young people attending Aboriginal primary health care services in Australia. BMC Infectious Diseases. 2015;15:370. Assessed on 26 August 2015.

27. Walker J, Tabrizi SN, Fairley CK, Chen MY, Bradshaw CS, Twin J, et al. Chlamydia trachomatis incidence and re-infection among young womenbehavioural and microbiological characteristics. Plos One. 2012;7(5):e37778.

28. Oakeshott P, Kerry S, Aghaizu A, Atherton H, Hay S, Taylor-Robinson D, et al. Randomised controlled trial of screening for Chlamydia trachomatis to prevent pelvic inflammatory disease: the POPI (prevention of pelvic infection) trial. BMJ. 2010;340:c1642. 